\title{
Retrospective Drug Use Evaluation of Gentamycin Use in Ambo Hospital, Oromia Region State, West Showa, Ethiopia
}

\author{
Belayneh Kefale Gelaw,, Gobezie Temesgen Tegegne ${ }^{1}$, Amsalu Degu Defersha ${ }^{1}$ and Alemitu Legese ${ }^{2}$ \\ ${ }^{1}$ Department of Pharmacy, College of Medicine and Health Science, Ambo University, Oromia region, Ethiopia \\ ${ }^{2}$ Ambo General Hospita Ambo, Ethiopia
}

\begin{abstract}
Drug should be used appropriately, safely and only when needed $50 \%$ or more of drug expenditure may be wasted through irrational prescribing, dispensing and patient use of drugs. Drug use evaluation is one of the useful methods to evaluate the rational use of drug in health institution and has performance of improved method that focus evaluation and improving medication use process with the goal of patient outcome. The objective of this study will to evaluate the rational use of gentamycin in Ambo Hospital. Retrospective systematic study was used to assess rational use of Gentamicin. The study was conducted by reviewing medication records of 400 patients, who received Gentamicin during hospitalization in Ambo general Hospital from Jan.1, 2013-Jan, 1, 2014. A systematic sampling method was used to select prescriptions in this hospital with Gentamicinand patient cards were located based on the medical record number on the prescription papers. Data was collected by using structured format and evaluated against WHO criteria for drug use evaluation as per standard treatment guideline of Ethiopia.Most patients were dosed as $5 \mathrm{mg} / \mathrm{kg} /$ day (59\%). The duration of therapy was found to be high in the range $2-7$ days $(64.25 \%)$. Gentamicin was mainly used as pneumonia treatment $(36 \%)$. Maintenance fluids were the most commonly co-administered medications with a frequency of $67.75 \%$. The use of Gentamicin was appropriate only in 189 cases $(47.25 \%)$ for the justification of use. Most of inappropriate uses were seen in terms of duration during treatment of pneumonia followed by frequency for the treatment of AGE. Consistency of prescriber to the national standard treatment guideline was found to be low.To improve rational use and prevent the development of resistance; prescribers should adhere to the national standard treatment guideline. Intensification of short-term trainings and antibiotic control systems are some of the possible solutions the hospital has to do.Gentamicin therapy does not meet the current STG of Ethiopia.
\end{abstract}

Keywords: Drug Use Evaluation, Gentamicin, Ambo

\section{INTRODUCTION}

Drugs are among the most expensive input of the health service. Drugs should be used appropriately, safely and only when needed. Good drugs use management is compulsory in health care system as drugs are the input that saves life. Most leading courses of death and disability in developing countries can be prevented. Treated or at least alleviated with cost effective essential drugs. $50 \%$ or more of drugs expenditure may be wasted though irrational prescribing, Dispensing and patient use of drugs. ${ }^{1}$

Pharmaceuticals can constitute up to $40 \%$ of the heath care budget in a developing
DOI: $10.5530 /$ ijopp.7.2.4

Address for correspondence: Dr. Belayneh Kefale Gelaw Department of Pharmacy, College of Medicine and Health Science, Ambo University, Oromia Region, Ethiopia

Mobile: +251913805290 E-mail: belayneh.kefale@ yahoo.com

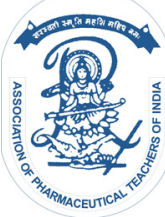

www.ijopp.org 
country. Yet large proportion of the population often lack essential medication. Because of its considerable impact on the quality of care and the cost of treatment. the selection of medicine is one of the most cost effective approaches to improving access to health care both in developing and developed countries. ${ }^{1}$ Anti biotic represent approximately 30\% of acute care hospitals drug expenditure they are prescribed for $20-50 \%$ of inpatients and their irrational use contribute to the emergency of resistant micro -organisms. Survey done in Switzerland has shown that $22-65 \%$ antibiotic prescriptions are either inappropriate or incorrect. ${ }^{2}$

Drug use evaluation (DUE) is an ongoing systematic process designed to maintain the appropriate and medication data before. During and after dispensing in order to assure appropriatetherapeutic decision making and positive patient outcome. ${ }^{3}$

Drug use evaluation is one of the useful methods to evaluate the rational drugs use in health institution and has a performance of improvement methods that focuses on evaluation and improving medication use processes with the goal of optimal patient outcome. Of medication use processes (prescribing preparing dispensing administering and monitoring) or specific outcome. $^{4}$

The discovery and use of antimicrobial agents have brought a major break through in therapy. A lot of previously intractable infectious conditions have now become amenable to antimicrobial therapy. Various classes of antibiotics have been discovered and used with varying degrees of success. Among these are the Aminoglycoside. Older members of this group, like the gentamicin, have been available for the treatment of urinary tract infections. However, the limited use of this drug coupled with the rapid development of resistant strains became a problem. ${ }^{5}$

Aminoglycoside antibiotics are frequently used as empirical antibiotics in critically ill patients against suspected gram-negative sepsis or in combination with others to cover some gram positive species. They bind to ribosomal subunits within bacteria, preventing protein synthesis, but the exact mechanism of action remains unclear. ${ }^{6}$

Gentamicin is synthesized by Micromonospora, a genus of Gram-positive bacteria widely present in the environment (water and soil). To highlight their specific biological origins, gentamicin and other related antibiotics produced by this genus (verdamicin, mutamicin, sisomicin, netilmicin, retymicin) generally have their spellings ending in $\sim$ micin and not in $\sim$ mycin. Gentamicin is a bactericidal antibiotic that works by binding the $30 \mathrm{~S}$ subunit of the bacterial ribosome, interrupting protein synthesis.
Gentamicin is an aminoglycoside antibiotic composed of a mixture of related gentamicin components and fractions and is used to treat many types of bacterial infections, particularly those caused by Gram-negative organisms. ${ }^{2}$ However, gentamicin is not used for Neisseria gonorrhoeae, Neisseria meningitidis or Legionella pneumophila. Gentamicin is also ototoxic and nephrotoxic, with this toxicity remaining a major problem in clinical use. ${ }^{7}$

Like all aminoglycosides, when gentamicin is given orally, it is not systemically active. This is because it is not absorbed to any appreciable extent from the small intestine. It is administered intravenously, intramuscularly or topically to treat infections. It appears to be completely eliminated unchanged in the urine. Urine must be collected for many days to recover all of a given dose because the drug binds avidly to certain tissues. E. coli has shown some resistance to gentamicin, despite being Gram-negative. Reluctance to use gentamicin for empirical therapy has led to increased use of alternative broad-spectrum antibiotics, which some experts suggest has led to the prevalence of antibiotic-resistant bacterial infections by MRSA and other so-called "superbugs". 6

Gentamicin is one of the few heat-stable antibiotics that remain active even after autoclaving, which makes it particularly useful in the preparation of some microbiological growth media. It is used during orthopedic surgery when high temperatures are required for the setting of cements (e.g. hip replacements). ${ }^{7}$

Evaluation of gentamicin use is therefore critical to controlling the emergence of resistant strains as well as cutting down of unnecessary expenditures and also ensuring that patients derive maximum benefit from its use.

\section{Statement of the Problem}

Drug use evaluation (DUE) is an authorized. Structured ongoing review of physicians prescribing Pharmacist dispensing and patient use of medications. It involves a comprehensive and after the mediation is dispensed. In managed health care system in identifying and integrating problem and making medication. ${ }^{3}$ In a retrospective analysis of 1,578 antimicrobial prescriptions at the institution's emergency room complex in 1989, prescribed antibiotics were considered inappropriate in $65.2 \%$ of cases and $61 \%$ were given prophylactic ally. ${ }^{8}$

In most developing countries governments spend about $20-50 \%$ of their national budget on drugs and medical sundries (World Bank, 1994). This makes the financial impact of pharmaceuticals on their economies to be substantial. Governments are thus concerned about the rational use of this drugs. ${ }^{4}$ 
Furthermore, the World Health Organization Policy Perspective on Medicine (2002) also indicates that even when drugs are made available, more than fifty percent are prescribed, dispensed or sold inappropriately while $50 \%$ of patients fail to take the medicines correctly resulting in harmful consequences.

One of the major consequences of such inappropriate use of antibiotics is the development of resistance strains of the hitherto susceptible organism. Inappropriate treatment could also lead to the ultimate; death of the patient. Indeed the WHO report on infectious diseases 2000 indicates that "without proper treatment gastro enteritis is a serious and frequently occurring disease that kills majority of the infected individuals. ${ }^{2}$

\section{Significance of the study}

Indealing with health problem like in appropriate use of drug that has impact on economy of the county, impalement of drug use evaluation program has useful to monitor in health care system.

The most challenging in our world today is the development resistance to most drug s specially antibiotic due to inappropriate use of drug which may leads treatment failure and imposable to treatment infection disease. To avoid such problem drug use evaluation is the most important. Drug has been used irrationally that reduce quality of patient care, was the resource and cause harm to patient. Because this due avoid medication related problem and increase the patient outcome.

The study design was to evaluate the use of gentamycin in ambo hospital to provide an overview of gentamycin use in hospital and to promote the rational prescribing, dispensing an administration of gentamycin. Hence, to reduce the emergency of antibiotic resistance. Moreover the study contribute in identifying medication related problem and areas of in appropriate use by that it help in identifying areas in which further information and education may be needed by health providers.

\section{OBJECTIVES}

\section{General objectives}

The general aim of this study was to evaluate gentamicin use atAmbo Hospital.

\section{Specific objectives}

- To analyses the pattern of gentamycin use among patient categories identified by age.

- To identify the illnesses most frequently treated with gentamicin.
- To determine whether gentamicin was appropriately prescribed in respect of dose, dose frequency, and dose duration.

- To identify areas in which further information and education was needed by health care provider.

- To evaluate reason for stopping (discontinuous)the gentamycin is based on guide line or not.

- To assess whether the indication of gentamycin as standard guideline or not.

\section{MATERIAL AND METHODS}

\section{Study Area and period}

A study was conducted from February to April 2014 in Ambo general hospital. Ambo is the capital city of west shwea zone of Oromia region state and bordered on the south by Gosokora, on the West by Awaro, on the north by Dobi and on the East by Awaro. The North Ambo is one of the Fastest growing towns in the region and has a total population of 64,513; male are 32,275 and female 32,238 living in 3 kebeles the total area of the town is 858,753 hectors the town has one referral hospital one MCH Special clinic and two health centres in addition to many other private facility

\section{Study Design}

Retrospective study design was conducted to evaluate the use of gentamicin in Ambo General Hospital. The criteria used for antibiotic selection include the following gentamicin with risk those that were not being controlled by antibiotic prescribing restriction system. Drug use evaluation was performed by reviewing patient medication records.

\section{Source of Population}

Medication records of patients who received gentamycin during hospitalization in Ambo General Hospital from Jan 1, 2013 to Jan 1, 2014.

\section{Sampling Techniques}

The study population was sampled by using systematic sampling to select representatives of the population. Sampling was started from separating prescription for the period of March to May 2014 then the patient prescriptions containing gentamycin for this period were counted. Then the card number of patients from prescriptions containing gentamicin was randomly chosen. Then from the card number every some calculated number was taken to sample the study populations. The card number was used to locate the patient medication records. 


\section{Sample Size determination}

It was suspected that $95 \%$ confidence interval was desired to estimate the proportion within $5 \%$ and the sample size drawn as(18).

$\left.\left.\mathrm{N}=\mathrm{Z}^{2} \times \mathrm{p}(1 \mathrm{p}) / \mathrm{W}^{2}=1.96\right)^{2}(0.5 * 0.5) / 0.05\right)^{2}$

$$
\mathrm{N}=384
$$

Where,

$\mathrm{N}=$ required sample size

$\mathrm{Z}=$ is multiplier for $95 \%$ confidence interval based on the normal distribution

$\mathrm{p}=$ expected prevalence

$\mathrm{W}=$ desired absolute precision $(5 \%)$

The above sample was going to be taken from a relatively small population $(<10,000)$, then the sample size has been adjusted as;

$\mathrm{NF}=\mathrm{n} /(1+(\mathrm{n} / \mathrm{N}))$,

$=384 /(1+(384 / 4000)=351$

\section{Data collection Instrument}

The data was collected by using open filling in structured format and the data were recorded in the format from the patient medication records.Four WHO criteria's for drug use evaluation (DUE) was used in this study; namely,

- Indication for use

- Dosage

- Frequency

- Duration to evaluate gentamicin use.

\section{Variable}

\section{Dependent variable}

- Indication of gentamycin

- Drug -drug interaction with gentamycin
- Side effect of gentamycin

- Dose frequency of gentamycin

- Gentamycin contra indication

- Duration of treatment

\section{Independent Variable}

- Age

- Sex

- Diagnosis

\section{Data Processing and Analysis}

The data collected was checked, coded and entered into Microsoft Excel and descriptive statistic was utilized to summarize the data. For this analysis p-value less than 0.05 considered as significant whereas $\mathrm{p}$-value greater than 0.05 considered asnon-significant. All statistical analyses were done manually using scientific calculator.

\section{Data quality Control}

The clarity and completeness checking of data collection format were under taken beforethe actual data collection and data clearing was done every day. Format without full information wasexcluded from the study.

\section{Ethical Consideration}

Formal letter written by department of pharmacy was given to ambo hospital in order to get permission to conduct the study. The confidentiality of the patient in patient medical record was maintained throughout the study time

\section{RESULTS}

A total of 400 patient's card were reviewed and analyzed for the use of gentamicin in Ambo general hospital. Among 400 patients, 191(47.7\%) were female and 209 $(52.25 \%)$ male. Most of them were under the age of less than five years (Table 1).

\begin{tabular}{ccc}
\hline Table 1: Age Distribution of Patients Included in the Study. & \\
\hline Age (years) & Numbers of patient card & $\%$ \\
\hline$<5$ & 276 & 69 \\
$6-16$ & 83 & 20.75 \\
$>16$ & 41 & 10.25 \\
\hline Total & 400 & $100 \%$ \\
\hline
\end{tabular}

Age distribution was biological classification neonatal, infant, child and adults 
Table 2: Demographic (sex) distribution of patient included in the study

\begin{tabular}{ccc} 
SEX & Number & \% \\
\hline Male & 209 & 52.25 \\
Female & 191 & 47.75 \\
\hline Total & 400 & 100 \\
\hline
\end{tabular}

\section{Table 3: Gentamicin daily dosing distribution}

\begin{tabular}{cccc}
$\begin{array}{c}\text { DAILY } \\
\text { Dosage(gmlday) }\end{array}$ & $\mathbf{5 m g l k g}$ day & $\mathbf{7 . 5 m g l k g}$ & $\mathbf{8 m g l k g}$ \\
\hline Frequency & 236 & 127 & 37 \\
Percent & 59 & 31.75 & 9.25 \\
\hline
\end{tabular}

Mostly, gentamycin was given $5 \mathrm{mg} / \mathrm{kg}$ day which covered $59 \%$ and the rest were $7.5 \mathrm{mg} / \mathrm{kg}$ and $8 \mathrm{mg} / \mathrm{kg}$ (Table 3).

\section{Table 4: Duration of gentamicin therapy distribution}

\begin{tabular}{ccc} 
Duration in days & Numbers of patient & $\%$ \\
\hline Stat & 14 & 3.5 \\
1 & 18 & 4.5 \\
$2-7$ & 257 & 64.25 \\
$8-14$ & 3 & 0.75 \\
No duration & 108 & 27 \\
\hline Total & 400 & $100 \%$ \\
\hline
\end{tabular}

About $64.25 \%$ was given for patients for the period of $2-7$ days, followed by no duration. But the list percentage was $0.75 \%$ which showed gentamycin was administered for long times for few cases.

\begin{tabular}{ccc}
\hline Table 5: Frequently co-administered drugs with gentamycin & \\
\hline DRUG & FREQUENCY & $\%$ \\
\hline MF & 271 & 67.75 \\
Ampicillin & 223 & 55.75 \\
Metronidazole & 1 & 0.25 \\
Ceftriaxzone & 48 & 12 \\
Procin-pencillin & 123 & 30.75 \\
Cry-pencillin & 3 & 0.75 \\
CAF & 2 & 0.50 \\
\hline Total & 400 \\
\hline
\end{tabular}

\begin{tabular}{|c|c|c|c|c|c|}
\hline Assessment & Total & Appropriate & $\%$ & Inappropriate & $\%$ \\
\hline Sepsis & 114 & 94 & 82.4 & 20 & 17.5 \\
\hline pneumonia & 144 & 50 & 34.7 & 94 & 65.5 \\
\hline SAM & 50 & - & - & - & 100 \\
\hline UTI & 8 & 5 & 62.5 & 3 & 37.5 \\
\hline Pertenats & 14 & - & - & 14 & 100 \\
\hline Meningitis & 10 & 10 & 100 & - & - \\
\hline AGE & 30 & - & - & 30 & 100 \\
\hline Joint Infancy & 10 & 10 & 100 & - & - \\
\hline Appendicitis & 20 & 20 & 100 & - & - \\
\hline Total & 400 & 189 & 47.25 & 211 & 52.75 \\
\hline
\end{tabular}

Gentamycin is the most prescribed appropriately for meningitis (100\%) and the least is for UTI (62.5\%) 


\begin{tabular}{ccc}
\multicolumn{2}{c}{ Table 7: Distribution of in appropriate use of gentamycin based on due criteria } \\
\hline DUE-CRITERIA & Number of error & $\%$ \\
\hline Duration & 94 & 44.5 \\
Frequency & 47 & 22.27 \\
Dose & 40 & 18.95 \\
Indication & 30 & 14.2 \\
\hline Total & 211 & 100 \\
\hline
\end{tabular}

In Ambo Hospital the duration, frequency, dose and indication of gentamycin was evaluated retrospectively. The most common indication of gentamycin was sepsis and pneumonia followed by SAM, appendicitis, age and others are showed in table 6.

\section{DISCUSSION}

In this study $59 \%$ of patient took $5 \mathrm{mg} / \mathrm{kg}$ daily and $31.75 \%, 9.25 \%, 7.5 \mathrm{mg} / \mathrm{kg}$ and $80 \mathrm{mg} / \mathrm{kg}$ daily according to standard treatment guide line for different cases it is better.

In this study the mean duration of gentamycin therapy was found to be 7 days and high in the range 2-7 days $(64.25 \%)$ followed by 1 days $(4.5 \%)$ which is better than the study done in Bahrainover extended duration of treatment $(2.7 \%)$ were encountered irrational drug therapy in infant with prescribing error is apparent in primary care practices which may be related to a lack of drug information $(9,11)$.

This study is showed that $52.75 \%$ of gentamicin is prescribed inappropriately. From this error duration (44.5\%), frequency $(22.27 \%$ ) accounts high percentage. It is close to the study done on antibiotic in Holland that show $22-65 \%$ antibiotic prescriptions were either inappropriate or in correct. $(3,10)$

Among the medication co administeredwith gentamicin fluid took first place with frequency of $271(71.5 \%)$ followed by ampicillin injection 223 (58.1), procaine penicillin $123(32 \%)$, ceftriaxone 48 (12.5), crystalline penicillin $3(0.78 \%)$ and metronidazole $1(0.3)$. In this study drug-drug interaction was not occurred b/c the prescribed the drugs as STG. In contrast the study done in Bahrain showed that $2.4 \%$ interaction with drugs.

In this finding majority of the patients use gentamycin for SAM (100\%), joint infection $(100 \%)$ appendicitis $(100 \%)$, meningitis $(100 \%)$, sepsis $(82.4 \%)$, pneumonia $(65.5 \%)$, UTI $(62.5 \%)$ which is in line of with standard treatment guideline of Ethiopia but patients use for acute gastritis $30(100 \%)$, UTI $(37.5 \%)$ were used inappropriately related to indication, dose, frequency. It is comparable with the study done in Nigeria and presented to WHO in 2002 by surveyors, $60 \%$ of antibiotics were presented unnecessary. ${ }^{10}$

Most of the in appropriate use was involved during prescription of gentamycin for pneumonia $65.3 \%$ and sepsis $17.5 \%$ with duration and frequency, respectively. It is comparable with the study undertaken in Nigeria and presented to WHO in 2000 by surveyors, $60 \%$ of antibiotic were prescribed unnecessary. ${ }^{10,12}$

Inappropriate use of medicine results waste of resourcesfor the patient, and result significant patient harm in term of poor patient out come and adverse drug reaction. In this finding the dosing pattern of gentamycin as therapy in the hospital was found that the dose prescribed was in appropriate in the patient of $30.85 \%$ and frequency of the drug prescribed $39.4 \%$. This show that dose error is not a common problem regarding to gentamicin prescribed in ambo hospital when it compares with the study done in Bahrain 54.1\% of prescription with omission error length of therapy was not states, in $43.5 \%$ of prescription with omission error dosing frequency $(20.8 \%)$ and dose strength $(17.7 \%)$ related error were most common regarding to irrational prescribing drug. ${ }^{11,13}$

In this study the use of gentamicin was found to be appropriate for the justification of use in 189 (47.25\%) and in appropriate in 211 (52.75\%). The value which is appropriate use of gentamycin in the study area is in line with the value obtained in Nepal which is $50 \%$.

The appropriate use of gentamicin in the current study is not in agreement with the retrospective study done in Sudan, $20 \% .^{12}$

The differences might be due to the fact that Ambo general hospital may how lack of controlling agents like drug therapeutic committee and lack of equipped with sufficient professionals as well as shortage of in service training for health professionals.

The use of gentamicin was found to be high for the treatment of pneumonia 144 (36\%) followed by sepsis, SAM, AGE, appendicitis, perforates, meningitis\& UTI in descending orders.

The mean duration of gentamicin therapy is 7 days and high in the range of 2-7 days followed by no duration, staff dose, one day and 8-14 days in descending orders. 
This is lower than the study done in Khartoum. This is an important factor as the number of days in which gentamicin used correlates with resistance prevalence. Additionally, this may indicate the prevalence of diseases in which gentamicin was used in the range mentioned above. ${ }^{11}$

\section{CONCLUSION}

Drug use evaluation of gentamycin with respect to indication, duration of therapy and frequency of administration manipulates correct prescription practice.

In Ambo general hospital as to WHO set criteria prescribers were not sticking to Ethiopian national STG.

The majority of in appropriateness was seen with duration of therapy. Results of the present study showed that inappropriate use of gentamycin was high which paves for the emergency of bacterial strains that are resistant to the available antimicrobial agent which in turn lead to increase in cost of therapy and treatment failure.

\section{RECOMMENDATIONS}

To improve the correct use of gentamycin in ambo hospital, the followingrecommendations were given:

- In a few patients, inappropriate dose and frequency needs to prescribed, so the prescriber should give attention when prescribe the gentamycin in Ambo Hospital.

- Easy access of the national STG to all health professionals setting continuous drug use evaluation programs.

- Hospital should have drug therapeutic committee (DTC) that can evaluate in correct prescription.

\section{ACKNOWLEDGMENTS}

We are very grateful to our college staff members for unreserved guidance and constructive suggestions and comments from the stage of proposal development to this end. We would like to thank Ambo University for supporting the budget which was required for this research. Finally, our deepest gratitude goes to Ambo Hospital staff workers who helped and allowed us to collect data from the hospital.

\section{Acronym and abbreviation}

DTC: Drug Therapeutic Committee

DUE: Drug use evaluation

DUR; Drug utilization review

UTIS;Urinary tract infection

HIV; Human immune virus

STG: Standard treatment guideline

Funding: Ambo University

Conflict of interest: None declared

Ethical approval: Approval and permission was sought from Ethical Review Board of College of Medicine and Health Sciences of Ambo University

\section{REFERENCES}

1. Olft A.J, Suttenfield J.L, Benzen C.S. MSH managing drug supply second edition, USA Kumarian, 1997; 4 (11): 122

2. Gunton V, Troillet N, Beney J, Boubaker K, Liithi C.J, Taffe P, et al. Impact of inter displinary strategies on antibiotic use in three hospital. Switzerland. J. antimicrobial chemo therapy. 2004:55 (3); 362-66.

3. The academy of managed care pharmacy; concept in managed care pharmacy Drugus evaluation Was hingt on Accessed online at http:Ildepts. washington.edulexpharmd ExpharmD DUE. HTML ON 612।2010

4. America society of hospital pharmacist. ASHP guidelines on the pharmacist's role in drug use evaluation.AMJ. Hospital. 1988: 45; 103-04.

5. Threlfall, E.J., Ward, LR. Decreased Susceptibility to ciprofloxacin in Salmorellaenterica serotype Typhi, United Kingdom. Emerg Infect Dis. 2001: 7: 448-50

6. Gopal. V, Keane. M and Ail. D. Survey on the use of gentamicin and vancomycin in adult intensive care units. JICS. 2013: 14 (3).

7. Moulds, Robert and Jeyasingham, Melanie. Gentamicin: a great way to start. Australian Prescriber. 2010: (33): 134-35.

8. Goljan, Edward F. Rapid Review Pathology 3rd ed. Philadelphia, PA: Elsevier. 2011: p. 241. ISBN 978-0-323-08438-3

9. Akande M.T, Ologe, O.M.Prescription patter at asecondary health care facility in IIrin ,Nigeria .Journal of Africa medicin. 2007: 4; 186-89.

10. Nicholas Z, Gordon J. Inlfuecing antibiotic prescribing in genralpractic. Africa of presciberfeed back and management guideline.1999.

11. Alkhaja K, Alansari T, Damanhori A, Sequerira R. Evaluation of drug utilization and prescribing error in infant; a primary care prescription based on study. Mddle Eas J. health policy 2007. 8; 350-57.

12. Abdelmumin IA. Investigation of drug use in health center in Khartum stat Sudan medical J. 1999. 37; 21-26.

13. Gentamicin spectrum of bacterial susceptibility and Resistance. Retrieved 15 May, 2012. 